\title{
HIGH SPEED VIDEOMETRIC MONITORING OF ROCK BREAKAGE
}

\author{
J. Allemand ${ }^{1}$, M. R. Shortis ${ }^{2 *}$ and M. K. Elmouttie \\ ${ }^{1}$ Institute of Geodesy and Photogrammetry, ETH Zurich, Zurich, Switzerland - jon_allemand@live.com \\ ${ }^{2}$ School of Science, RMIT University, GPO Box 2476, Melbourne, Victoria 3001, Australia - mark.shortis@rmit.edu.au \\ ${ }^{3}$ CSIRO Energy, PO Box 883, Kenmore, Queensland 4069, Australia - marc.elmouttie@csiro.au
}

\section{Commission II, WG II/5}

KEY WORDS: Rock breakage, Comminution, High speed video, Stereo-photogrammetry

\begin{abstract}
:
Estimation of rock breakage characteristics plays an important role in optimising various industrial and mining processes used for rock comminution. Although little research has been undertaken into 3D photogrammetric measurement of the progeny kinematics, there is promising potential to improve the efficacy of rock breakage characterisation. In this study, the observation of progeny kinematics was conducted using a high speed, stereo videometric system based on laboratory experiments with a drop weight impact testing system. By manually tracking individual progeny through the captured video sequences, observed progeny coordinates can be used to determine 3D trajectories and velocities, supporting the idea that high speed video can be used for rock breakage characterisation purposes. An analysis of the results showed that the high speed videometric system successfully observed progeny trajectories and showed clear projection of the progeny away from the impact location. Velocities of the progeny could also be determined based on the trajectories and the video frame rate. These results were obtained despite the limitations of the photogrammetric system and experiment processes observed in this study. Accordingly there is sufficient evidence to conclude that high speed videometric systems are capable of observing progeny kinematics from drop weight impact tests. With further optimisation of the systems and processes used, there is potential for improving the efficacy of rock breakage characterisation from measurements with high speed videometric systems.
\end{abstract}

\section{INTRODUCTION}

Mining of natural resources has been a key financial contributor to the economies of countries that have large reserves of minerals and ores. With finite quantities of these natural resources, compounded with increased competition by various multi-national mining companies, there is pressure to improve the efficiency of the mining process, thus increasing production and minimising costs. One significant cost in the mining of minerals and ores is the energy cost to break rocks down to a size where the metal or compound can be separated. The process of breaking down rock particles to smaller fragments is commonly referred to as comminution.

The characterisation of comminution tests is crucial for accurate determination of rock breakage characteristics. Analysis of the comminution tests will assist in the optimisation of various industrial and mining processes, such as rock crushing machinery and rock blasting technologies. Most comminution tests are focussed predominantly on determining how various input energies and forces transfer through to rock breakage characteristics. A primary indicator are the size and velocity distributions of rock particles from the breakage, known as progeny.

There are a variety of comminution tests currently utilised to determine the particle characteristics for different types of rock (Bearman etal., 1997; Mwanga, et al., 2015; Zhang and Zhao 2013). Mwanga et al. (2015) provides a thorough analysis of the current methods by comparing them against set criteria for a geo-metallurgical testing program to determine the efficiency and quality of the tests. The review provides an overview of each of the tests, the rock characteristics for which they are used to define and a discussion on how each test compares to the criteria set for the review. Within the range of testing options, a very common technique to determine the rock breakage characteristics is the drop weight test. As the name suggests, a heavy weight or driven hammer is used to fracture the rock sample.

Zhang and Zhao (2013) also discusses various tests to understand different rock mechanics behaviour. This paper provides a detailed description of each testing process, the parameters estimated and how each type of test has evolved. The paper highlights the extensive use of high speed photography systems for quantitative purposes and proposes that these systems should be investigated further to better understand rock fragmentation behaviour.

A common focus in comminution analysis is the segmentation of the resulting particulate matter and the creation of an optimal size distribution for the next stage of processing (Bearman et al., 1997; Fandrich et al., 1998; Jemwa and Aldrich, 2012; Mwanga et al., 2015; Noy, 2013; Sanchidrián et al., 2008; Zhang and Zhao, 2013). Active stereo photogrammetric and laser scanning systems have been successfully used to automate the process of estimating particle size distributions of ore piles travelling on a conveyor belt. These techniques have the potential benefit of reducing the power intensive, mechanical systems currently used to estimate particle distributions (Jemwa and Aldrich, 2012; Noy, 2013).

A fundamental understanding of different rock parameters and characteristics related to the comminution of rocks is essential in order to optimise processing machinery, minimise power consumption for desired particle sizes and in turn reduce costs. 
The main outcome of drop weight testing for comminution purposes is estimation of likely particle size distributions (Bearman et al., 1997). The development of an automated measurement of particle distributions with a high-speed 3D photogrammetric system of the tests can reduce the time and labour required to estimate the rock breakage characterisation compared to using traditional techniques.

The use of photogrammetry systems is well proven for measuring static or dynamic particulate matter. Maas and Gruen (1995) presents a multi-camera system and a least squares matching method for 3D particle tracking velocimetry. Spreafico et al. (2017) analysed the deposition from rock bridge fractures using low altitude photography from a drone. More closely related to comminution tests, Kim et al. (2015) utilises a combination of 2D and 3D photogrammetric techniques along with simulations to achieve consistent results for determining coefficients associated with rock fall trajectories down a slope. Finally, there are instances where comminution tests have been undertaken using high speed photography to make 2D measurements of dynamic fragmentation events (Ma et al., 2011; Shuaeib et al., 2004).

The review of the current literature has identified that there is considerable potential in the use of photogrammetry to better understand rock breakage characterisation. Accordingly, research was conducted for an honours level project (Allemand, 2016) to test the use of a high speed videometric system for 3D analysis of drop weight tests, with the aim to observe progeny kinematics.

\section{MEASUREMENT SYSTEM COMPONENTS}

\subsection{Cameras}

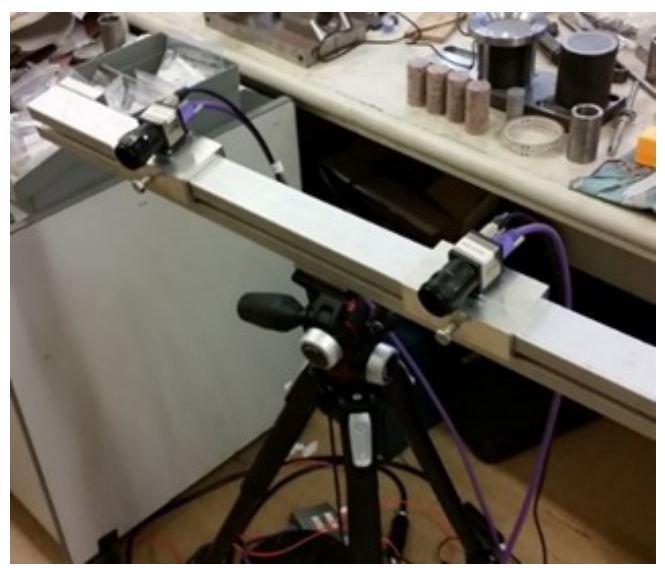

Figure 1. IDS cameras on the base bar.

The high speed videometric system used for this research is a stereo IDS camera system (IDS, 2018) comprising two IDS UI3060CP-C-HQ machine vision cameras fitted with $16 \mathrm{~mm}$ lenses (see Figure 1). These cameras feature a CMOS colour sensor, global shutter, maximum resolution of 1936 by 1216 pixels, 5.86 micrometre pixel spacing and a maximum frame rate at full resolution of 166 frames per second. For the data capture, the cameras are connected via a combination of USB 3.0 cables and fibre optic extension cables to individual ports of a custommade PC. The PC has two $250 \mathrm{~GB}$, high speed, solid state drives (SSDs), so that the video sequences are recorded to separate SSDs to ensure uninterrupted capture.
The cameras are synchronised using a hard wired trigger pulse. Test videos taken of bouncing balls and preliminary drop weight tests indicated that there was no discernible delay between the captured pairs of frames and there would be no significant systematic errors incurred in the particle tracking.

\subsection{Capture, Processing, Photogrammetric and Modeling Software}

StreamPix is a software package developed by NorPix for the acquisition and control of multiple cameras (NorPix, 2018). This software was used to capture the synchronised pairs of videos from the IDS system. The videos are recorded directly to the SSDs without decoding the Bayer pattern to ensure all frames are recorded within the available band width of the interfaces. StreamPix captures the videos in a native file format which is then exported to an uncompressed AVI format. The Bayer pattern is decoded within the software to enable the AVI video to be recorded and viewed in colour.

To record a set of videos, the physical pulse trigger button is pressed to start the live feed of the cameras to the PC. The software, based on predetermined settings, then begins synchronous data recording once record is pressed within the software. The same button is then pressed to stop the recording.

Xvid is a free and open-source video codec that can be used for high quality compression of video (Xvid, 2018). The uncompressed AVI files output by StreamPix, particularly of the calibration process, were deemed too large to manage efficiently and so Xvid was used for the compression of the files. The highest quality setting was used to minimise the impact of compression artefacts.

The Vision Measurement System (VMS) research software was used for photogrammetric processing (Geometric Software, 2018). VMS has the capability to compute multi-camera selfcalibration networks with base length and rotation angle relative orientation constraints, as well as process image sequences using network or resection-intersection solutions based on video or still images. A utility program enables the computation of the mean relative orientation of the stereo-cameras based on all constrained pairs of images included in the self-calibrating network. The derived camera calibrations and mean relative orientation are then used to process the image sequences.

In this case the $3 \mathrm{D}$ measurements extracted from the video sequences were computed using a straightforward intersection solution using left and right image measurements from the stereo-camera system. For this project, VMS was used to read in the compressed AVI files output from the recording processes and acquire manual measurements of rock particles on a frame by frame basis. Estimated locations in 3D are based on a coordinate system with the origin located at the mid-point between the two camera perspective centres; the $\mathrm{Z}$ axis represents the depth to the target; the $\mathrm{X}$ axis is parallel to the base between the two camera perspective centres and $\mathrm{Y}$ axis is orthogonal.

MATLAB, produced by MathWorks (MathWorks, 2018) was used to design and implement code for automation, computation and visualisation of the data obtained from the high speed photogrammetric systems. The software was used to derive information and plots from the output 3D Cartesian coordinates obtained from VMS. 


\subsection{Impact Testing Facility}

The Instron Dynatup 9250g machine (see Figure 2) is located at the CSIRO Rock Cutting Laboratory, Queensland Centre of Advanced Technologies and is designed for impact testing of various materials. It is similar in design to impact testing facilities utilised by the JKTech Laboratory Services for their proprietary JK Drop Weight Testing which provides industry with sample ore breakage characteristics. It is fully computer controlled, including data capture during the impact event. It has a maximum drop height of $1.25 \mathrm{~m}$ and weight of $80.5 \mathrm{~kg}$ which allows for a maximum input energy of 1010 Joules at a maximum velocity of $5.0 \mathrm{~m} / \mathrm{s}$. It is housed within a clear protective casing with a guided hammer and anvil, which supports observing the particle kinematics from rock comminution. The test section inside the barrier casing is approximately $500 \mathrm{~mm}$ by $500 \mathrm{~mm}$ by $100 \mathrm{~mm}$, but the useable volume and visibility is limited by the hammer support structure and cables.

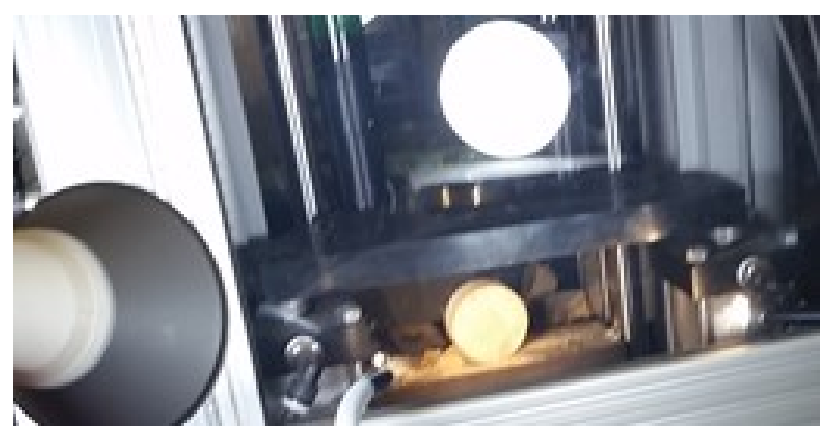

Figure 2. Impact testing machine, sample and lighting set up.

\section{EXPERIMENT METHOD}

\subsection{Experiment Design}

The comminution experiments involved placing a steel anvil and the sample at the base of the test machine, onto which a $20 \mathrm{~kg}$ flat hammer head would strike the sample. This was enclosed within the protective barrier with a matte black card placed at the back of the enclosure to ensure the fragmented particles had sufficient contrast against other objects within the case (see Figure 2). The experiments used core samples of sandstone rock that were approximately $60 \mathrm{~mm}$ in diameter and ranged in width from $20 \mathrm{~mm}$ to $35 \mathrm{~mm}$.

The tests were conducted indoors with no natural light, so additional lighting was required to account for the decreased exposure time of the cameras when recording at high frame rates. A dual-pronged fibre optic lighting system provided much of the light onto the sample within the protective barrier of the test machine. Two additional flood lights were used to provide additional lighting to ensure the particles would be visible on the highest frame rate setting of the cameras.

The dual-pronged lights were positioned equally on either side of the line of sight from the camera to the sample as not to obstruct the field of view or have significant light reflecting off the protective barrier where the comminution event was expected to occur. As can be seen in Figure 2, these lights were placed practically against the protective screen to minimise the reflected light but to ensure the rock sample was illuminated as much as possible.
As the only access in and out of the test machine was via the hinged front panel, the cameras had to be placed at a sufficient distance away to ensure each sample could be placed within the closure and the aligned with the hammer without disturbing the cameras. The fibre optic lights were placed on a support with the prongs bent such that the device could be moved out of the way to enable ready access into the impact test machine but allow replicate conditions and reduce the time required to reset between tests.

The lighting arrangement was sufficient to allow acceptable exposures at a rate of 390 frames per second. At this frame rate the resolution of the cameras is reduced to 640 by 480 pixels, but the small field of view required for the test sample compensated for the loss of resolution. The faster frame rate ensured that very rapid particle motion could be captured.

The two cameras were set up in a classic stereo configuration in front of the drop weight test machine, making use of a rigid base bar mounted on a heavy duty tripod to ensure stability (see Figure 1). The cameras were fixed onto the base bar with bolts and the lenses were taped to ensure a consistent relative orientation and camera calibrations. The fields of view of the cameras are shown in Figure 3.

The base bar was levelled to provide an approximate vertical reference. However line of sight restrictions caused by components of the test machine required the cameras to view the sample at an oblique angle from above (see Figure 5) and limited the base separation to $350 \mathrm{~mm}$. To obtain the field of view shown in Figure 3, the range between the cameras and the test object was set at $1600 \mathrm{~mm}$, resulting in a base to distance ratio of 1 to 4.6
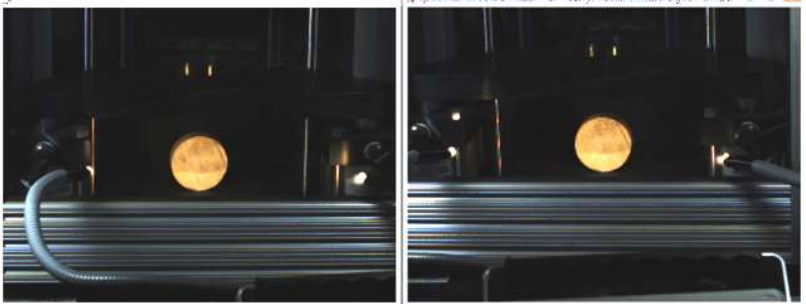

Figure 3. Fields of view for the two cameras.

\subsection{Camera Calibration}

Camera calibrations were carried out to ensure the accuracy of the stereo measurements and to simultaneously determine the relative orientation of the cameras. It is well understood that a transparent sheet will affect the line of sight between the cameras and the test sample, so calibrations were carried out with a test board in front of and behind the barrier to estimate the effects of the additional distortion. A physical parameter set of principal point, principal distance, radial and decentring distortions was used in both cases, under the assumption that the additional distortions from the barrier would be absorbed by the parameter set (Shortis, 2015).

For the sake of expediency during transport and manipulation behind the barrier, A2 and A4 size planar boards with a random pattern of 38 white targets, both coded and non-coded, on a matt black surface were used for the calibrations. The limitations of a 2D calibration object (Boutros et al., 2015) were accepted on the basis that stringent accuracy was not a priority 
for the experimental work. The A2 board was used for calibration in front of the barrier, but the field of view was such that only part of the board could be seen by both cameras. The A4 board had to be cut down to fit within the sample area of the test machine, which reduced the number of visible targets (see Figure 4).

Video sequences were captured of the boards being rotated and moved around within the fields of view of the cameras. The maximum duration of the recordings, based on the available capacity of the SSDs at 390 fps and 640 by 480 resolution, is several minutes. Consequently, calibration sequences in front of the barrier required only a single video capture to ensure that around 40 different perspectives of the calibration boards could be acquired. Rotating and re-positioning the calibration board behind the transparent barrier proved to be a cumbersome and slow process, so the system was used in individual frame capture mode rather than continuous video capture.

The calibration network in front of the barrier comprised 45 images from each camera and all 38 targets. The calibration set inside the transparent barrier was limited to 40 images from each camera and only 17 targets. In each case the board was moved around and rotated within the fields of view, but the amount of variation behind the transparent barrier was much more restricted. The networks were processed with a relative orientation constraint for the left and right cameras.

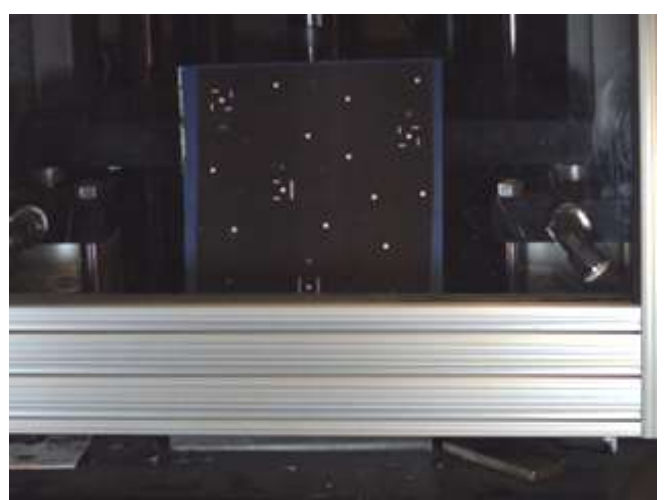

Figure 4. Calibration board positioned inside the impact test machine.

\subsection{Impact Test Video Capture}

Three tests of sandstone core rock samples were conducted. Before each test, the sample was carefully aligned with the hammer and the anvil. The enclosure door was closed and hammer was raised up ready for release. The lighting was shifted back into position and a live feed of the cameras was checked to ensure the sample was clearly visible in the fields of view. The video recording was then initiated and the hammer was released. The video recording continued until all progeny particles had come to rest.

The video sequence was trimmed to only include the period of interest defined by the motion of the hammer and progeny. This was undertaken to minimise file sizes before the export to uncompressed AVIs onto the main storage disk. The SSDs were then erased and the impact test machine cleaned of all fragmented particles ready for subsequent tests to be completed.

\subsection{Data Processing}

The first step in the data processing was to compress the AVI files using the Xvid codec and a dual pass to ensure that all frames were included. This was particularly important for the calibration sequences due to the uncompressed file sizes of approximately $1 \mathrm{~Gb}$

VMS requires still images to process the calibrations. The left and right synchronised frames can be manually identified in each video stream for the two cameras. To improve the efficiency of the processing, a MATLAB script was written to automatically capture images from video sequences. Based on a desired number of frames and identification of the two video files, the script extracts the paired frames and generates TIFF image files.

VMS automatically recognises the coded targets on the calibration boards, computes a resection of each frame and then back-drives to all remaining targets. Three networks are then computed. The first uses an externally constrained solution and fixed calibration to obtain good estimates of the camera orientation parameters. The second solution uses a selfcalibration with a free network to ensure that all target coordinates are unconstrained and determined with the most favourable precision. The final solution introduces the relative orientation constraints to ensure that the initial estimates of the base separation and relative rotation angles are as close as possible to the final estimates. From previous experience, poor starting values for the relative orientation parameters can lead to many spurious image measurement rejections or a non-global minimum for the network.

The stereo-measurements were then captured from the synchronised AVI video files, based on the camera calibrations and relative orientation derived from the calibration network with the target board inside the barrier. To align the coordinate system with the gravity vector, manual measurements were taken of the front face of the sample prior to hammer impact. The orientation and centroid of this face was used to perform a rigid body transformation of the stereo-camera coordinate system to the test sample coordinate system (see Figure 5).

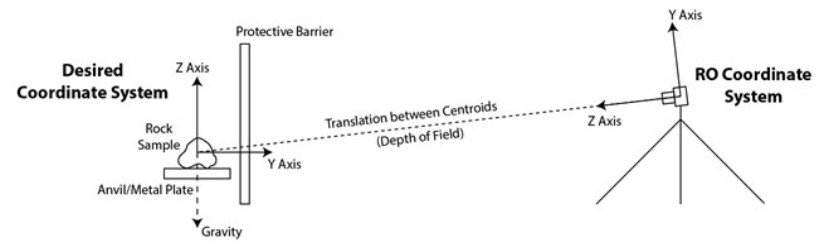

Figure 5. Coordinate system transformation.

To measure the fragmented particle trajectories, image observations of each particle in both left and right video frame were performed. This process involved identifying a particle in both images, using a cross-hair measurement tool to create a zoomed region window on the particle of interest, and then mouse-clicking a best estimate of the particle centroid. Figure 6 shows a pair of image frames with measured particles in both perspectives and a zoomed region of interest showing the particles and the exact measurement location.

This process was repeated multiple times for a single particle over the sequence of frames. Care was taken to estimate the centre of the particle and often the frames had to be stepped back and forward to ensure that the same particle was being 
observed. Additionally, particles that bounced around the enclosure were avoided as these are not representative of the kinematics of interest for fragmented particles trajectories after comminution.

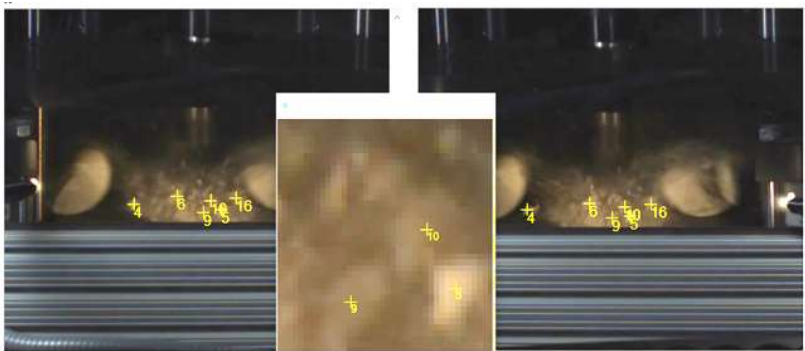

Figure 6. Manual measurement of progeny particles.

Two types of least squares curve fit were used to model the trajectories of the progeny particles. The first type is an XYZ curve that assumes the particles to be following a curved path in all three dimensions:

$$
f\left(x y z_{i}\right)=\left\{\begin{array}{l}
f\left(x_{i}\right)=a_{x_{i}} t^{2}+b_{x_{i}} t+c_{x_{i}} \\
f\left(y_{i}\right)=a_{y_{i}} t^{2}+b_{y_{i}} t+c_{y_{i}} \\
f\left(z_{i}\right)=a_{z_{i}} t^{2}+b_{z_{i}} t+c_{z_{i}}
\end{array}\right\}
$$

This approach was expected to produce a better fit to spinning particles. A simpler, second approach of a $\mathrm{Z}$ curve assumes that the particles are travelling in a straight line in the $X Y$ dimensions, but a curved path in the $\mathrm{Z}$ coordinate:

$$
f\left(x y z_{i}\right)=\left\{\begin{array}{c}
f\left(x_{i}\right)=b_{x_{i}} t+c_{x_{i}} \\
f\left(y_{i}\right)=b_{y_{i}} t+c_{y_{i}} \\
f\left(z_{i}\right)=a_{z_{i}} t^{2}+b_{z_{i}} t+c_{z_{i}}
\end{array}\right\}
$$

Particle velocities were computed using the Euclidean distance travelled, divided by the 2.5 millisecond time between frames. It was deemed that any bias introduced by the linear motion assumption would be negligible compared to other sources of error, particularly the manual measurements of the images.

\section{RESULTS AND ANALYSIS}

\subsection{Camera Calibration and Relative Orientation}

The results of the camera calibration networks for outside and inside the transparent barrier are shown in Table 1. The effect of the transparent barrier of the impact test machine is significant with more than $100 \%$ degradation of the RMS image error. The effect on the precisions of the target coordinates is less but still very significant. The primary factor in the degradation is the uncompensated distortions caused by the refractive surfaces, however loss of contrast due to attenuation through the transparent material of the barrier will also influence the results through increased noise.

Table 1. Camera calibration results.

\begin{tabular}{|l|c|c|}
\hline Value & $\begin{array}{c}\text { Outside } \\
\text { Barrier }\end{array}$ & $\begin{array}{c}\text { Inside } \\
\text { Barrier }\end{array}$ \\
\hline Number of exposures & 90 & 80 \\
\hline Number of targets & 38 & 17 \\
\hline RMS image error (pixels) & $1 / 27$ & $1 / 12$ \\
\hline $\begin{array}{l}\text { Mean precision of target } \\
\text { XYZ coordinates (mm) }\end{array}$ & 0.012 & 0.017 \\
\hline
\end{tabular}

A comparison of selected camera calibration parameters for camera 1 is shown in Table 2. Similar results were obtained for camera 2. Clearly there are significant changes to the principal point location and principal distance.

Table 2. Selected camera calibration parameters for camera 1.

\begin{tabular}{|l|c|c|c|}
\hline Value & $\begin{array}{c}\text { Outside } \\
\text { Barrier }\end{array}$ & $\begin{array}{c}\text { Inside } \\
\text { Barrier }\end{array}$ & $\begin{array}{c}\text { Significant } \\
\text { Difference? }\end{array}$ \\
\hline Principal point X (mm) & 0.098 & 0.041 & Yes \\
\hline Principal point Y (mm) & -0.141 & -0.053 & Yes \\
\hline Principal distance $(\mathrm{mm})$ & 16.333 & 15.945 & Yes \\
\hline $\begin{array}{l}\text { Radial distortion at } 2 \mathrm{~mm} \\
\text { radius (microns) }\end{array}$ & -8.5 & -10.7 & No \\
\hline
\end{tabular}

The effects of multiple media and refractive surfaces on closerange photogrammetric measurement is well documented for underwater systems for habitat mapping (Shortis, 2015) and laboratory experiments such as water tanks (Maas, 2015). The optical path through the refractive interfaces must be modelled or, as is the case here, absorbed by the standard camera model. According to Snell's Law, a plane parallel sheet will cause an apparent displacement of objects behind the barrier that increases with incidence angle. This effect is rotationally symmetric and should be partly absorbed by small increases in the principal distance and the radial lens distortion component of the calibration model. However, the effect of refraction invalidates the assumption of a single projection centre for the cameras (Sedlazeck and Koch, 2012), which is the basis for the physical parameter model, and thereby introduces uncompensated errors which must be absorbed by the image measurement residuals.

Contrary to expectations, Table 2 suggests small decreases in the principal distance and the radial lens distortion component. This unexpected result can be attributed to the very poor geometry of the calibrations and the narrow field of view covered by the array of targets in each case.

To investigate the effects of a transparent barrier in more controlled and comparative circumstances, a separate experiment was conducted. The IDS stereo camera system was calibrated with and without a thin, transparent acrylic sheet between the cameras and an A3 calibration board. The networks comprised a total of 80 exposures and 38 targets with a strongly convergent geometry and good coverage across the entire fields of view of the cameras. In this instance there were small increases to the principal distances and radial lens distortion profiles as expected, but at levels well below the threshold of significance. Similar to the results for the impact testing machine networks, there were significant changes to the principal point location and the RMS image error degraded by a factor of 2.4 times. This separate test was considered to be a more valid demonstration of the refractive effects of a transparent, planar barrier.

The effect of the transparent barrier on the relative orientation parameters is shown in Table 3. All parameters demonstrate a significant difference. In the separate experiment with the acrylic sheet, there were some small but significant changes to the base length and rotations. Similar to the results in Table 2 for the impact test machine barrier, the base length between the cameras increased for the network with the acrylic sheet in place. 
Table 3. Relative orientation parameters.

\begin{tabular}{|l|c|c|c|c|}
\hline & \multicolumn{2}{|c|}{ Outside Barrier } & \multicolumn{2}{c|}{ Inside Barrier } \\
\hline Parameter & Camera 1 & Camera 2 & Camera 1 & Camera 2 \\
\hline $\begin{array}{l}\text { Base } \\
(\mathrm{mm})\end{array}$ & -172.9 & 172.9 & -179.3 & 179.3 \\
\hline $\begin{array}{l}\text { Omega } \\
\text { (degrees) }\end{array}$ & 0.02 & -0.02 & 0.28 & -0.28 \\
\hline $\begin{array}{l}\text { Phi } \\
\text { (degrees) }\end{array}$ & -4.33 & 7.25 & 1.23 & 12.71 \\
\hline $\begin{array}{l}\text { Kappa } \\
\text { (degrees) }\end{array}$ & 0.50 & 0.40 & 0.07 & 0.64 \\
\hline
\end{tabular}

However the poor geometry of the calibration networks for the impact testing machine prevent any confident conclusions being drawn. Further, there is an assumption here that the stereocamera system was undisturbed during the calibration and measurement phases of the impact tests. This cannot be guaranteed and could be a possible source of the large change in the phi rotations between the video capture for the outside and inside calibrations. Reference targets on the body of the impact testing machine would have provided a more definitive check mechanism, however a visual comparison of fixed features in the frames from the calibration and measurement sequences suggests that there was no disturbance to either camera.

\subsection{Stereo Measurements}

The manual measurements of the edge of the core sample were used as a test of the precision and accuracy of the stereo system. The core sample is a cylinder with a diameter of $60 \mathrm{~mm}$. The estimated radius of the circular profile of the sample from the image measurements was $30.05 \mathrm{~mm}$ with an RMS error of $0.99 \mathrm{~mm}$.

The RMS error of the circular fit correlates well with the estimated precisions of the $\mathrm{XYZ}$ coordinates of the progeny particles. The estimated precisions of the $\mathrm{X}, \mathrm{Y}$ and $\mathrm{Z}$ coordinates were in the ranges of $0.53-0.72 \mathrm{~mm}, 0.36-0.40 \mathrm{~mm}$ and 3.1-4.2mm respectively. The large disparity between the $X$, $\mathrm{Y}$ plane and the $\mathrm{Z}$ depth precisions is a consequence of the relatively poor base to distance ratio of 1:4.6 for the stereo measurement system.

The precision in the depth direction could be improved by a more favourable base to distance ratio. However, it should be noted that industrial comminution machinery is not designed for optimal camera geometries to observe progeny kinematics and the camera geometry must be designed around the machinery. The alternative of additional cameras to provide different perspectives would theoretically provide improved precision and also greater reliability. Nevertheless the restrictions on fields of view remain and there is a penalty in terms of more complex logistics and greater processing demands.

The tracking of progeny after comminution was carried out based on operator identification of the particles across the frames in the video sequences. Measurement of particle centroids is a labour-intensive process with multiple issues identified for this technique.

To determine the coordinates of each progeny in each frame, rough approximations were made visually in each left and right image of the specific progeny. Although this method was simple, it has multiple sources of error. First, it relied on the ability of the operator to approximate the centroid of the individual progeny, which relies on the proper illumination, focus and contrast of the particle in the imagery. Figure 6 shows frames where significant shadowing and poor contrast makes the process of particle identification difficult, particularly for a sample with homogeneous texture and colour. Due to the direction of cameras and lighting, progeny in the background projecting away from the cameras are difficult to observe either from shadowing or occlusion from progeny in the foreground. This impact was clear in the spread of tracked progeny shown in Figures 7 and 8 .

To identify unique progeny between left and right images, the operator had to scroll back and forth through the sequence trying to visually track and identify an individual particle. Larger particles were generally easier to identify and track, but the precision which the centroid was determined in each image decreased considerably, thus likely adding significant noise to progeny trajectories.

Another key point of error that can be introduced with the manual centroid identification is from non-uniformly shaped particles and the rotation of the progeny as it travels along the trajectory. This has a potential to introduce a bias or oscillation into the observed trajectory due to incorrect centroid determination.

\subsection{Progeny Trajectories}

MATLAB was used to generate displacement vector diagrams. The particle trajectory coordinates and the vectors between one frame and the next show the displacement of the particles (see Figure 7). The cylindrical sample shown in the figure was included to verify the datum transformation and demonstrate that the plotted displacement vectors are correct with respect to the starting location of the sample.

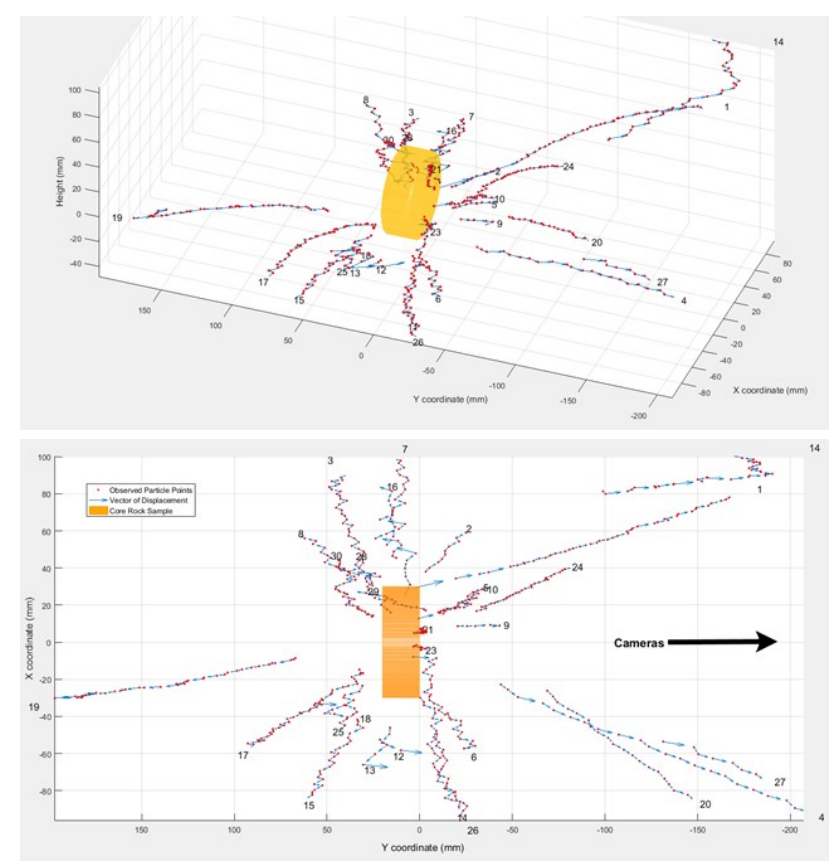

Figure 7. Displacement vectors of the progeny particles perspective (top) and plan (bottom). 
The vectors clearly show that the data is noisy, and the variation is greatest in the $\mathrm{Y}$ coordinate of the plot, which corresponds primarily to the $\mathrm{Z}$ coordinate of the stereo photogrammetric system. The 'saw tooth' effect is clear evidence of the greater uncertainty of the stereo measurements in the direction of depth from the cameras.

Figure 8 shows the particle coordinates and trajectories modelled using least squares curve fitting. The XYZ and Z curve fits (see Equations 1 and 2) are shown along with the particle locations. The majority of the progeny appear to follow vertical parabolic arcs away from the sample location as expected. In most cases the progeny follow a constant bearing and the $\mathrm{XYZ}$ and $\mathrm{Z}$ curve fits overlay each other consistently. In some cases there are clear differences between the two types of curve fit, generated by gross errors, collisions and the spin of large particles. The differences in the curve fit results can be used as a detector to identify unusual behaviour for further investigation.

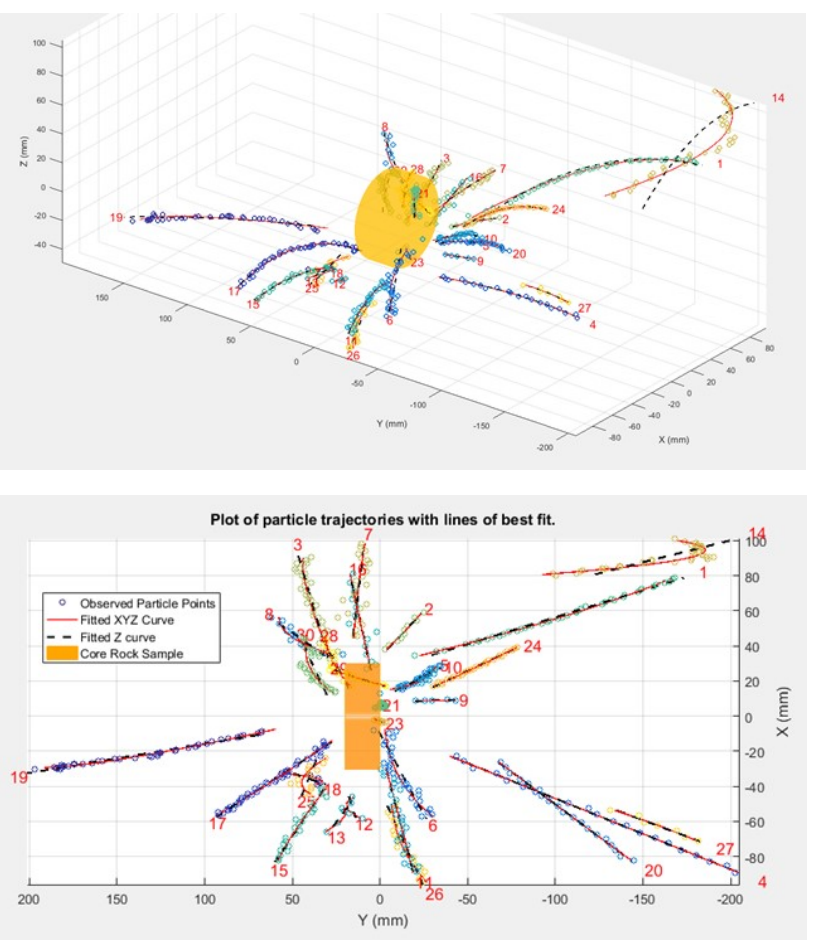

Figure 8 . Trajectory curve fits of the progeny particles perspective (top) and plan (bottom)

\subsection{Progeny Velocities}

The distribution and change of the progeny velocities is shown in Figure 9. The velocities are derived from the $\mathrm{Z}$ curve fit given as equation (2), similar results were obtained using the XYZ curve fit. The solid black line represents the mean velocity of the progeny at each frame and the thick red line represents the linear regression. The mean velocity shows a consistent decrease from frame to frame. The frame with the most progeny tracked is highlighted in blue. The mean progeny velocity for this frame was $1.2 \mathrm{~m} / \mathrm{sec}$ with a standard deviation of $1.0 \mathrm{~m} / \mathrm{sec}$.

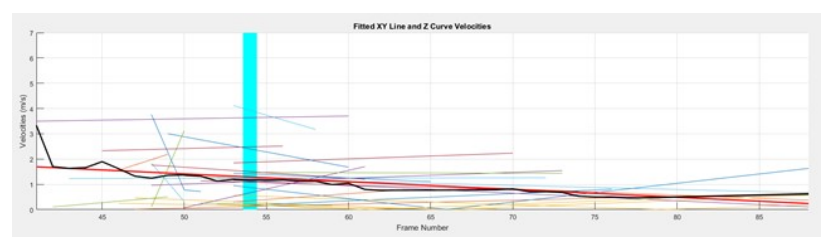

Figure 9. Progeny velocities plotted against frame number.

The method for determining progeny velocities is quite crude and could be improved for more reliable results. An algorithm for smoothing the velocities over multiple frames may be better suited to reduce the noise in the determined velocities. The small sample size, restricted by manual measurement and selection of well-defined particles, could lead to bias in the results. Nevertheless the linear regression shows a strong correlation which suggests relatively reliable results.

\section{CONCLUSIONS}

This research successfully implemented a high speed videometric system in a laboratory experiment of drop weight testing, from which progeny kinematics could be determined. Through data processing and analysis of results, the research succeeded in showing the trajectories and velocities of progeny could be derived following comminution of the rock sample.

Several key factors were identified from this research for optimisation and improvement of the high speed photogrammetric systems and methods used for observing progeny kinematics. Some of these were:

- provision of effective lighting and selecting suitable frame acquisition rates to improve the quality of the images,

- $\quad$ increasing the number of cameras and perspectives used to potentially improve precision, reliability and correct progeny identification,

- modelling systematic biases from protective barriers to improve observation precisions, and

- inclusion of fixed reference targets within the field of view to ensure reliability of the system.

However, two major issues remain to be resolved. First, the manual image measurement process is tedious and prone to error. Straightforward improvements such as image matching from three or four cameras would provide a more efficient, reliable and accurate process, but would of course still be limited by image quality and foreground obstruction factors. The second issue is the estimation of progeny size, which is an important factor in the effectiveness of the system to provide the information required by rock engineers. A potential solution to this demand is to model each progeny in $3 \mathrm{D}$, based on multiple perspectives and the rotation of the particle. The combination of image matching and 3D modelling would produce a structure-from-motion solution that should be able to estimate the sizes of a significant sample of progeny.

Overall, this research has provided a strong proof-of-concept for the potential for high speed photogrammetry to improve the efficacy of rock breakage characterisation. Further development of the measurement and identification techniques will lead to a more efficient and comprehensive solution that can include the distribution of progeny size, as well as trajectories and velocities. 


\section{ACKNOWLEGDEMENTS}

Dr Xing Li and Mr Craig Harbers (CSIRO) are acknowledged for their assistance with the experiments conducted in the CSIRO Rock Cutting Laboratory. Dr Sarma Kanchibotla and Mr Farhad Faramarzi (JKMRC) are acknowledged for their expert advice regarding comminution testing.

\section{REFERENCES}

Allemand, J., 2016. Investigation of high speed photogrammetry for rock breakage characterisation. Unpublished Geospatial Major Project Report. RMIT University. 134 pages.

Bearman, R. A., Briggs, C. A. and Kojovic, T., 1997. The application of rock mechanics parameters to the prediction of comminution behaviour. Minerals Engineering, 10(3): 255-264.

Boutros, N., Shortis, M. R. and Harvey, E. S., 2015. A comparison of calibration methods and system configurations of underwater stereo-video systems for applications in marine ecology. Limnology and Oceanography: Methods, 13(5): 224236.

Fandrich, R. G., Clout, J. M. F. and Bourgeois, F. S., 1998. The CSIRO Hopkinson Bar Facility for large diameter particle breakage. Minerals Engineering, 11(9): 861-869.

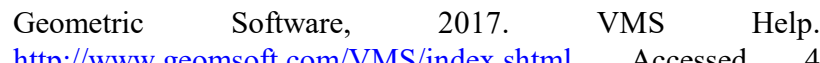
Accessed 4 January 2018 .

IDS, 2018. Imaging Development Systems GmbH https://en.ids-imaging.com Accessed 4 January 2018.

Jemwa, G. T. and Aldrich, C., 2012. Estimating size fraction categories of coal particles on conveyor belts using image texture modeling methods. Expert Systems with Applications, 39(9): 7947-7960.

Ma, S. P., Yan, D., Wang, X. and Cao, Y. Y., 2011. Damage observation and analysis of a rock Brazilian disc using highspeed DIC method. Applied Mechanics and Materials, 70: 8792.

Maas, H.-G. and Gruen, A., 1995. Digital photogrammetric techniques for high-resolution three-dimensional flow velocity measurements. Optical Engineering, 34(7): 1970-1976.

MathWorks, 2018. MATLAB - The Language of Technical Computing. https://au.mathworks.com/products/matlab/ Accessed 4 January 2018.

Mwange, A., Rosenkranz, J. and Lamberg, P., 2015. Testing of ore comminution behaviour in the geometallurgical context - a review. Minerals, 5(2): 276-297.

NorPix, 2016. Multiple camera digital video recording software. https://www.norpix.com/products/streampix/streampix.php Accessed 4 January 2018.

Noy, M. J., 2013. Automated rock fragmentation measurement with close range digital photogrammetry. Measurement and Analysis of Blast Fragmentation, pp. 13-21.

Sanchidrián, J. A., Segarra, P., Ouchterlony, F. and López, L. M., 2008. On the accuracy of fragment size measurement by image analysis in combination with some distribution functions. Rock Mechanics and Rock Engineering, 42(1): 95-116.
Shortis, M. R., 2015. Calibration techniques for accurate measurements by underwater camera systems. Sensors, 15(12): 30810-30826; doi: 10.3390/s151229831.

Shuaeib, F. M. et al., 2004. Drop weight testing rig analysis and design. Pertanika Journal of Science and Technology Supplement, 12(2): 159-175.

Sedlazeck, A. and Koch, R., 2012. Perspective and nonperspective camera models in underwater imaging - overview and error analysis. Outdoor and large-scale real-world scene analysis. F. Dellaert, J.-M. Frahm, M. Pollefeys, L. Leal-Taixé and B. Rosenhahn, Springer Berlin Heidelberg. 7474: 212-242.

Spreafico, M. C., Franci, F., Bitelli, G., Borgatti, L. and Ghirotti, M., 2017. Intact rock bridge breakage and rock mass fragmentation upon failure: quantification using remote sensing techniques. The Photogrammetric Record, 32: 513-536. doi:10.1111/phor.12225

Xvid, 2016. This is Xvid. https://www.xvid.com/ Accessed 4 January 2018.

Zhang, Q. B. and Zhao, J., 2013. A review of dynamic experimental techniques and mechanical behaviour of rock materials. Rock Mechanics and Rock Engineering, 47(4): 14111478. 